\title{
Power Consumption Comparison for Regular Wireless Topologies using Fault-Tolerant Beacon Vector Routing
}

\author{
Luke Demoracski and D. R. Avresky \\ Northeastern University \\ Network Computing Laboratory \\ 360 Huntington Avenue, Boston, MA \\ ldemorac@ece.neu.edu, avresky@ece.neu.edu
}

\begin{abstract}
Fault-tolerant Beacon Vector Routing (FBVR) is an efficient technique for routing in the presence of node failures. Several common wireless topologies exist that can be used with this technique. This paper compares the power consumption of various regular topologies using FBVR and makes appropriate recommendations. The topology types include Mesh, Torus, Communication Graph, and F-Cycle Ring (FCR).

An existing analytical method for power consumption prediction is used. The results of this analytical method are compared against simulation results, which match closely, showing a high level of confidence in the power consumption results.
\end{abstract}

\section{Introduction}

In wireless sensor networks there is a need for topologies with low power consumption. Several topology types have been previously compared on the basis of throughput, latency, and greedy routing [12] success without fallback and flooding (FF) in [10]. Regular topology types include Mesh [2], Torus [13], F-Cycle Ring (FCR) [5], and Communication Graph [10]. However, this analysis [10] did not include power consumption.

In wireless sensor network applications, units may have limited battery life. Thus, comparison of their power consumption characteristics is of great use. The original contribution of this paper is to compare these topologies on the basis of power consumption, both with and without the fallback and flooding (FF) technique.

A previously applied power consumption approach [3] is modified for the topology comparison. This approach was used to analytically predict the power consumption of a given network and to compare that prediction against the simulation environment [3], to further validate the confidence of the results. Thus far, this approach has only been applied to Unit Disk Graphs and has not been applied to the regular topologies. The new contribution of this paper is the application of the power consumption approach to regular topologies.

The rest of this paper is organized as follows. Section 2 provides a brief introduction to BVR and clusterbased fault-tolerant BVR and describes the simulator assumptions. Section 3 describes the analytical power consumption model. Section 4 compares and contrasts the results for the topology types. Section 5 provides a conclusion and suggestions for future research.

\section{Fault-Tolerant Beacon Vector Routing}

The technique for Fault-Tolerant Beacon Vector Routing (FBVR) [8], is a fault-tolerant enhancement to the original Beacon Vector Routing (BVR) [12] technique.

The BVR technique in [12] is summarized as follows. BVR routes are based on minimum distance criteria. Each node maintains its distance from all $r$ beacon nodes in the network, represented as the position, $J(n)$ in Equation 1 . The distance from each beacon node $i$ to a given node $n$ is represented as $B_{i}(n)$.

With each packet hop, the distance function, $o(k, n, q)$ in Equation 2, of the surrounding nodes is compared against the current node, and the node with minimum distance function is selected. The distance function includes a weighting function, $w_{i}(n, q)$ in Equation 3, for the $k$ closest beacons. The weighting function provides a higher weighting to beacons that are closer to the destination, thus pulling the packets better toward their individual destinations. 


$$
\begin{array}{r}
J(n)=<B_{1}(n), B_{2}(n), \ldots, B_{r}(n)> \\
o(k, n, q)=\sum_{i \in C(k, q)} w_{i}(n, q) *\left|B_{i}(n)-B_{i}(q)\right| \\
w_{i}(n, q)= \begin{cases}10 & \text { if } B_{i}(n)>B_{i}(q) \\
1 & \text { otherwise }\end{cases}
\end{array}
$$

Greedy routing is attempted through BVR, using the criteria of Equation 2. Failed packets are retried through mechanisms of fallback and flooding (FF). Fallback sends a packet to the closest beacon to the destination, and then directly to the destination. If fallback fails, then flooding will send a packet to the closest beacon to the destination and then flood with the scope of the destination. The FF techniques are costly in terms of power consumption.

To improve routing success, and power consumption per successful packet, the fault-tolerant techniques are applied. Four techniques, known as Cluster-Based BVR (CBVR) [9], FBVR Type1, Type2, and Type3, further described in [8], combine to form a complete fault-tolerant approach CFBVR-Opt. CBVR applies clustering to reduce the number of hops to the destination. FBVR Type1 provides a dynamic reconfiguration technique, which forms a restoration tree around the failed nodes and corrects the local beacon vector information. FBVR Type 2 provides an improved weighting function that will more strongly pull the packets toward the destination. FBVR Type 3 violates the rule of Equation 2 and allows the packet to proceed for up to $H$ hops towards the best available distance function, as opposed to the minimum distance function. FBVR Type 3 avoids backtracking by maintaining the recent nodes visited in its header. The combination of these approaches, CFBVR-Opt, provides improved routing success, throughput, and power consumption, compared with BVR. Further BVR protocol descriptions are found in [8], [9], [12].

A simulator [10] is used for the various comparisons. Uniform traffic was used that transmits from any node to any other node in the network. Also, a Poisson distribution [4] for time between requests was used. In this distribution, the time for packet generation is $t=(1 / \lambda) * \ln (1-R)$, where $R$ is a random number between 0 and 1 . Further details of the framework of this simulator are found in [10].

\section{Power Consumption Model for Regular Topologies}

The power consumption model used is summarized in this section. Several existing power consumption models were previously investigated [6], [7], [11], [14], [15]. In order to predict the power characteristics of the regular topologies, a Markov Chain model [15] was modified to include the fault-tolerant protocol without flooding,
CFBVR-Opt, and the protocol with flooding but without fault-tolerance, CBVR-FF. A similar derivation was performed in [3], but only for the Unit Disk Graph topology, and not for the Torus, Mesh, FCR, and Communication Graph. The power consumption model [3] is a hybrid of [6] used for power consumption parameters and [15] used as a starting point for analytical prediction. The power consumption parameters closely follow the specifications of the TR 1000 radio transceiver of RF Monolithics used in [6].

Table 1 from [6] shows the state parameters used in the power consumption analysis. The RX state consumes power for a packet to be listened for at a given node, decoded, and interpreted. The TX state consumes power to transmit a packet from a given node. Note, TX and RX are not allowed to occur simultaneously for a given node [6]. The IDLE state consumes power when the node is actively listening for packets. The SLEEP state is a power-saving mode where the node is not actively listening for packets.

The analytical power consumption was measured by the following equations from [3]. Equation 4, $P_{A V}$ from [3], is the average per node power consumed for the network, obtained by summing the total power for each node $i, P_{t o t} i$, and dividing by the number of active nodes $n$. Equation 5, $P_{A V P S P}$ from [3], is the average power consumed per node per successful packet, obtained by dividing Equation 4 by the number of successful packets, $N_{S}$.

$$
\begin{array}{r}
P_{A V}=\left(\sum_{i=1}^{n} P_{t o t i}\right) /(n) \\
P_{A V P S P}=\left(\sum_{i=1}^{n} P_{t o t i}\right) /\left(n * N_{S}\right) \\
T_{\text {dist } i=m}= \begin{cases}T_{\text {dist }} & \text { if } m=1 \\
1 & \text { otherwise }\end{cases}
\end{array}
$$

Through derivation, using [15] as the starting point, the expected power consumption was predicted. A discrete time Markov Chain [15] is used to model the set of states in the network. Recursive equations to model the network activity were developed using dynamic programming in [3].

Through simulation profiling, the average per node transition probabilities were determined for the fallback and flooding (CBVR-FF) and fault-tolerant (CFBVR-Opt) techniques. The average remaining power cost per node from any given point in time in the network is the term $J()$ [3]. Equations for $J()$, derived and presented in [3], show the average remaining power cost per node for CFBVR-Opt and CBVR-FF respectively. A network congestion factor is applied to compensate for network congestion due to traffic patterns, navigation around failed nodes, and packet retries for fallback and flooding. The average packet transmission distance, $T_{\text {dist }}$ from [3], shown in Equation 6, is applied to 
A)

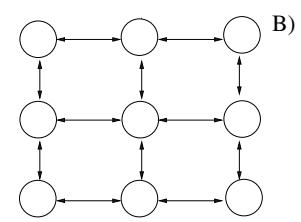

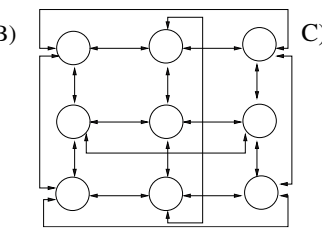
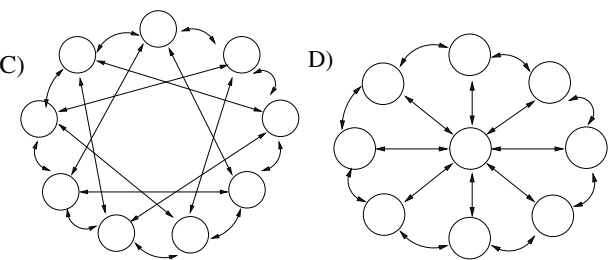

Figure 1. Topologies: (From Left to Right) A) Mesh, B) Torus, C) FCR, D) Communication Graph

the first iteration $m$ of the equations for $J()$ to account for the network size.

The prediction of the next state $j$ depends on the transition probability from state $i$ to state $j, q_{(i, j)}$. Tables 2 through 4 illustrate the $q_{(i, j)}$ values for the regular topologies, that were determined through simulation profiling in this paper. In each recursive iteration of $J()$, the average power cost remaining per node, is computed until either the battery runs out or all packets are sent. The battery strength is reduced by the associated power cost in Table 1 for the given state, and the packet buffer is reduced when a packet completes its transmission or is dropped. A packet can either succeed or be dropped, and associated routing success probabilities are applied in each case.

\section{Topology Comparison}

In [3], a power consumption model was presented to analytically predict the power consumption of wireless networks. In [3], only the Unit Disk Graph was analyzed, but in this paper, the Mesh [2], Torus [13], FCR [5], and Communication Graph [10] are considered, for comparison. These topology types are illustrated in Figure 1 from [10], and are described in further detail in [10].

The transition probabilities were profiled and are shown in Tables 2 through 4 for Mesh, Torus, and FCR topologies in the presence of 15 percent node failures, and each for a 625 node network. The $q_{(i, j)}$ values in Tables 2 through 4 represent the transition probability from any state $i$ to each of the four state types $j$. Power consumption results for the Communication Graph are not shown because it has negligible routing success in the presence of node failures [9].

The statistics in Tables 2 through 4 were obtained while the network was under saturation, in a stable state, for at least 1000 simulation clock cycles, and until the moving averages criteria in [4] were satisfied. Moving averages criteria are satisfied when successive data measurements differ by less than one percent, for five successive measurement windows [4]. Each transition was considered in each clock cycle, including transitions to the same state.

For the Mesh and Torus topologies, the FF technique incurs additional IDLE time and additional TX and RX time due to packet retransmissions. A significant amount

\begin{tabular}{|l|l|}
\hline State & Power Consumption $(\mathrm{mW})$ \\
\hline TX & 14.88 \\
RX & 12.5 \\
IDLE & 12.36 \\
SLEEP & 0.016 \\
\hline
\end{tabular}

Table 1. Power Consumption for each State

\begin{tabular}{|l|l|l|}
\hline $\begin{array}{l}\text { Probability of } \\
\text { Transition to } \\
\text { a Given State }\end{array}$ & $\begin{array}{l}\text { CFBVR-Opt } \\
\text { Probability } \\
\text { (percentage) }\end{array}$ & $\begin{array}{l}\text { CBVR-FF } \\
\text { Probability } \\
\text { (percentage) }\end{array}$ \\
\hline$q_{(i, R X)}$ & 22.6 & 23.1 \\
$q_{(i, T X)}$ & 22.6 & 23.1 \\
$q_{(i, I D L E)}$ & 17.5 & 17.8 \\
$q_{(i, S L E E P)}$ & 37.3 & 36.0 \\
\hline
\end{tabular}

Table 2. Mesh Transition Probability

\begin{tabular}{|l|l|l|}
\hline $\begin{array}{l}\text { Probability of } \\
\text { Transition to } \\
\text { a Given State }\end{array}$ & $\begin{array}{l}\text { CFBVR-Opt } \\
\text { Probability } \\
\text { (percentage) }\end{array}$ & $\begin{array}{l}\text { CBVR-FF } \\
\text { Probability } \\
\text { (percentage) }\end{array}$ \\
\hline$q_{(i, R X)}$ & 22.8 & 25.7 \\
$q_{(i, T X)}$ & 22.8 & 25.7 \\
$q_{(i, I D L E)}$ & 16.0 & 19.0 \\
$q_{(i, S L E E P)}$ & 38.4 & 29.6 \\
\hline
\end{tabular}

Table 3. Torus Transition Probability

\begin{tabular}{|l|l|l|}
\hline $\begin{array}{l}\text { Probability of } \\
\text { Transition to } \\
\text { a Given State }\end{array}$ & $\begin{array}{l}\text { CFBVR-Opt } \\
\text { Probability } \\
\text { (percentage) }\end{array}$ & $\begin{array}{l}\text { CBVR-FF } \\
\text { Probability } \\
\text { (percentage) }\end{array}$ \\
\hline$q_{(i, R X)}$ & 36.8 & 33.1 \\
$q_{(i, T X)}$ & 36.8 & 33.1 \\
$q_{(i, I D L E)}$ & 24.9 & 26.8 \\
$q_{(i, S L E E P)}$ & 1.5 & 7.0 \\
\hline
\end{tabular}

Table 4. FCR Transition Probability 
of SLEEP time occurs in Mesh and Torus for two reasons. First, failing packets do not travel completely to the destination, thereby providing congestion relief among certain nodes. Second, the Poisson variable $R$ and the Uniform traffic distribution result in some nodes having empty queues for short periods of time.

For the FCR topology, a small amount of time is spent in the SLEEP state because it has relatively short transmission distances, it handles more packets, and thereby has a more streamlined traffic pattern. Due to the short transmission distances required for FCR, the fault-tolerant CFBVROpt technique, which searches for additional paths using FBVR Type3, incurs a significant overhead in transmission distance. Also, fallback and flooding (FF) has little success for FCR. Thus, the FCR TX and RX times for CFBVR-Opt are higher than that for CBVR-FF.

The analytical power consumption model was compared against simulation model, and is shown in Figure 2-A,B,C.

The analytical model applies the profiled transition probabilities shown in Tables 2 and 4, and recursively computes the analytical power consumption equations that are derived in [3], for all transmitted packets, for the regular wireless topologies in this paper. This recursive computation continues until either the battery, $b$, runs out of strength, or all packets are transmitted. A battery strength of $3000 \mathrm{~mW}$ is used. In the technical literature, it is shown that cellular phone power ranges from $100 \mathrm{~mW}$ to $4000 \mathrm{~mW}$, thus 3000 $\mathrm{mW}$ is an acceptable battery strength estimation.

The simulation model was enhanced to measure energy characteristics for each of the topology types. At the beginning of time in simulation, all nodes are in the SLEEP state, and they change states to TX, RX, and IDLE depending on the traffic pattern and queue load. Packets are transmitted into the node queues using a Poisson distribution [4].

The predicted results closely match the simulation results. In all cases, the predicted results are higher overall because they reflect the worst case upper bound of potential network congestion situations. The simulation results are higher initially for all cases, during the first few hundred packets, because the network has not yet reached the saturated state, and several nodes are still in the SLEEP state consuming extra power. The simulation results follow a linear trend when saturation is achieved.

In the cases of Mesh and Torus, the power consumption of CFBVR-Opt is significantly less than that of CBVR-FF. This is because the additional retries of fallback and flooding are very costly and consume additional power. For the Mesh topology, Figure 2-B illustrates that CFBVR-Opt transmits the entire packet stream while CBVR-FF runs out of battery strength and still has packets left to be transmitted. For FCR in Figure 2-C, CBVR-FF consumes less power compared to CFBVR-Opt for two reasons: FF retries fail very quickly after they are attempted and fallback and flooding never succeed for FCR. The topologies of Mesh and Torus are more likely to be used than FCR, so in general CFBVR-Opt has better results than CBVR-FF.

Comparing Figure 2 between the topologies, FCR consumes the least power. However, FCR has much less routing success than Torus and Mesh, as shown in [10]. Both Torus and Mesh have very high routing success in the presence of failures [10]. Figure 2-A shows that Torus has significantly less power consumption than Mesh. This is because, Torus has smaller packet transmission distances, and thus smaller network diameter, than Mesh, and therefore consumes less power in its packet transmission. FCR has smaller transmission distance than Torus, and thus consumes less power than Torus, but at the expense of routing success.

The power consumed per node per successful packet, $P_{A V P S P}$ from Equation 5, was compared for the topologies and is shown in Table 5. The data for this measurement was collected when the network was in a stable state for at least 1000 clock cycles and when the moving averages criteria [4] was achieved.

\begin{tabular}{|l|l|l|}
\hline Topology & CFBVR-Opt & CBVR-FF \\
Type & $P_{A V P S P}(\mathrm{~mW})$ & $P_{A V P S P}(\mathrm{~mW})$ \\
\hline Torus & 1.048 & 1.733 \\
Mesh & 1.755 & 2.868 \\
FCR & 4.827 & 4.322 \\
\hline
\end{tabular}

\section{Table 5. Comparison of Power Consumption Per Node Per Successful Packet, $P_{A V P S P}$}

Table 5 shows that Torus consumes the least power per successful packet compared with the other topology types. Mesh consumes the next least power per successful packet, and FCR consumes a very large amount of power per successful packet. The routing success for FCR is much lower than that of Torus and FCR, as shown in [10]. Thus, the corresponding power per successful packet is higher for FCR.

For Mesh and Torus, the CBVR-FF technique requires additional packet transmissions, and has less routing success, which results in a higher $P_{A V P S P}$ compared to CFBVR-Opt. For FCR, the CBVR-FF technique has a smaller $P_{A V P S P}$ than CFBVR-Opt because the faulttolerant Virtual Search capability of CFBVR-Opt increases the packet transmission length to find alternative paths, and the transmission length is very small for the FCR topology type. Overall, the FF technique incurs a significant penalty in power consumed per successful packet with FCR being the exception case due to its small transmission distance.

Although Torus has excellent power consumption and is used in wireless applications [13], [16], Mesh is more realistic because wraparound transmission links in Torus are difficult to achieve. Mesh has in general been shown to be 

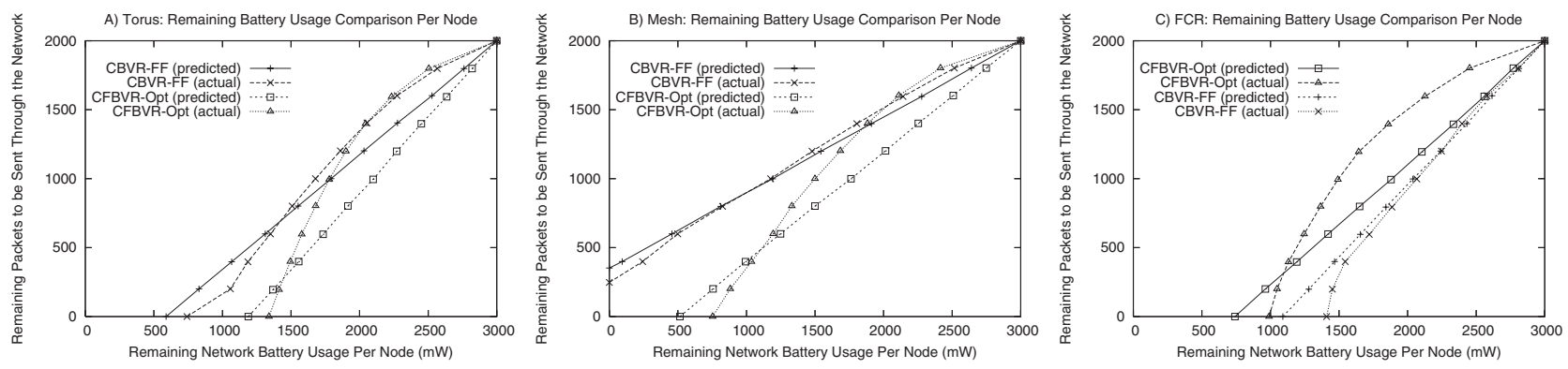

Figure 2. Examples of Battery Usage for 15 percent node failures, 625 node network, 2 percent beacons

an increasingly accepted topology in industry [1], [2]. Thus, Mesh is recommended as the preferred topology.

\section{Conclusion and Future Work}

In summary, the power consumption for the topology types of Mesh, Torus, FCR, and Communication Graph have been compared for BVR protocols. In the presence of node failures, Torus provides the least power consumption per successful packet, compared with the other topology types. However, Mesh is closest in behavior to the Torus, and is more realistic for deployment purposes. For both FCR and Communication Graph, although power consumption is low, the power consumption per successful packet is high, thus these topology types are not recommended.

As future work, the investigation of behavior of the proposed protocols at the MAC layer is being considered. Further topologies are being considered for analysis, including the smaller tree-like topologies of Multi-Slave Piconet and Scatternets in Bluetooth.

\section{References}

[1] Tropos networks, a technology whitepaper: Open standards for broadband wireless networks: Wi-fi and wimax. http://www.tropos.com, October 2004.

[2] Wi-mesh alliance: Wi-mesh proposal for ieee 802.11s. http://www.wi-mesh.org/, 2006.

[3] D. R. Avresky and L. Demoracski. Fault-tolerant adaptive beacon vector routing for ad hoc wireless networks. Submitted to the IEEE Transactions on Parallel and Distributed Systems, December 2005.

[4] D. R. Avresky, V. Shurbanov, R. Horst, W. Watson, L. Young, and D. Jewett. Performance modeling of servernet san topologies. Journal of Supercomputing, Kluwer Academic Publishers, 14(1), July 1999.

[5] D. R. Avresky and Y. Varoglu. Scalable and resilient to denial-of-service attacks overlay networks. In the Supplemental Proceedings of the International Conference on Dependable System and Networks - Workshop 3, July 2005.
[6] S. Basagni, A. Carosi, and C. Petrioli. Sensor-dmac: Dynamic topology control for wireless sensor networks. IEEE Vehicular Technology Conference, September 2004.

[7] B. Chen, K. Jamieson, H. Balakrishnan, and R. Morris. Span: An energy-efficient coordination algorithm for topology maintenance in ad hoc wireless networks. Wireless Networks, Kluwer Academic Publishers, 8:481-494, 2002.

[8] L. Demoracski. Fault-tolerant beacon vector routing for mobile ad hoc networks. In the Proceedings of the International Parallel and Distributed Processing Symposium - Workshop 16 on Dependable Parallel, Distributed and Network-Centric Systems, April 2005, Denver, CO.

[9] L. Demoracski and D. R. Avresky. Correctness of faulttolerant cluster-based beacon vector routing for ad hoc networks. In the Proceedings of the IEEE International Conference on Wireless and Mobile Computing, Networking and Communications, August 2005, Montreal, Canada.

[10] L. Demoracski and D. R. Avresky. Topology selection for fault-tolerant beacon vector routing in wireless sensor networks. In the Proceedings of IEEE International Conference on Networking and Services, October 2005, Tahiti.

[11] L. Feeney and M. Nilsson. Investigating the energy consumption of a wireless network interface in an ad hoc networking environment. Proceedings of Twentieth Annual Joint Conference on IEEE Computer and Communications Societies, April 2001.

[12] R. Fonseca, S. Ratnasamy, D. Culler, S. Shenker, and I. Stoica. Beacon vector routing: Scalable point-to-point in wireless sensornets. Intel Research, IRB-TR-04-012, May 2004.

[13] E. Kail, G. Nemeth, and Z. R. Turanyi. Throughput of ideally routed wireless ad hoc networks. In the Proceedings of ACM MobiHOC, 2001, Long Beach, CA, USA.

[14] C. Ma and Y. Yang. Battery-aware routing for streaming data transmissions in wireless sensor network. Second International Conference on Broadband Networks, October 2005.

[15] A. Markopoulou, Y. Li, C. Chan, and N. Bambos. Energyefficient communication in battery-constrained portable devices. Second International Conference on Broadband Networks, October 2005, Boston, MA.

[16] S. Panichpapiboon, G. Ferrari, N. Wisitpongphan, and O. K. Tonguz. Route reservation in ad hoc networks: Is it a good idea? In the Proceedings of IEEE WCNC, 2005. 